\title{
Modellierung und Bewertung der maritimen Leercontainerlogistik unter Berücksichtigung des Container- Poolings
}

\author{
Prof. Dr.-Ing. Nina Vojdani, Dipl.-Wirt.-Ing. Felix Lootz \\ Universität Rostock \\ Lehrstuhl für Produktionsorganisation und Logistik
}

\begin{abstract}
Vor dem Hintergrund zunehmenden Kostendrucks und der Suche nach Effizienzsteigerungen im Leercontainermanagement gewinnen zukünftig kooperative Gestaltungsansätze vermehrt an Bedeutung. Einen Ansatz zur kooperativen Neugestaltung des Leercontainermanagements in der maritimen Containerlogistik stellt die gemeinsame Nutzung eines Container-Pools durch mehrere Reedereien und Containerleasinggesellschaften dar. Dieses sogenannte Container-Pooling orientiert auf die Reduzierung des Repositionierungs-, Transport-, Umschlag- und Lagerungsaufwands durch gezielte Ausnutzung struktureller Ungleichgewichte. In der Praxis ist dieser Ansatz insbesondere aufgrund unternehmenspolitischer Gründe (Container als Werbeträger) bisher kaum umgesetzt. Für eine Optimierung und zur Unterstützung der operativen Planung sind mathematische Ansätze notwendig, die die verschiedenen Gestaltungsmöglichkeiten des Container-Poolings modellseitig berücksichtigen sowie zwischen Pool-, Reeder- und Leasing-Containern unterscheiden. Ziel dieses Beitrags ist daher die Vorstellung eines mathematischen Optimierungsansatzes, der sowohl die Abläufe der maritimen Containerlogistik beschreibt, als auch die Einsparungspotentiale durch den Einsatz des ContainerPoolings quantifiziert. Es wird gezeigt, dass durch den Einsatz des ContainerPoolings Kosteneinsparungen im Vergleich zum unkooperativen Verhalten vorliegen.
\end{abstract}

\section{$1 \quad$ Bedeutung der maritimen Leercontainerproblematik}

Der internationale Warenaustausch wurde in den 60er Jahren des vergangenen Jahrhunderts mit der Einführung normierter Seetransportbehälter revolutioniert und resultierte in einem technologischen Wandel der maritimen Transportlogistik. Die Implementierung von standardisierten Seecontainern führte dabei zu Etablierung weltweiter Containerliniendienste auf Basis von Containerschiffen und Containerterminals in den Seehäfen. Mit dem Einsatz wieder verwendbarer Transportbehälter geht im Allgemeinen eine anfänglich unbedeutende Problematik einher. Auf einen Vollcontainertransport folgt in der Regel die Repositionierung des Leercontainers. In den Seehäfen unterliegen Leercontainer damit vor dem Lasteinsatz und während des lastfreien Rücklaufs Transport-, Umschlag-, Lagerund Aufbereitungsprozessen. Dies bindet Ressourcen für den Vollcontainerumschlag und ist mitverantwortlich für ein zunehmendes Verkehrsaufkommen im Hafengebiet. Leercontainerprozesse zählen zu den Stützprozessen und erbringen kein geldwerten Kundennutzen. Die Kosten eines Leercontainertransports entsprechen aber nahezu 
denen eines Vollcontainertransports. Insbesondere im trimodalen Hinterlandverkehr sowie im maritimen Containerverkehr können die Kosten der Leercontainerlogistik die Anschaffungskosten übersteigen [Boi06].

Im Speziellen kann die durch den Einsatz wieder verwendbarer Container entstandene Leercontainerthematik anhand vielfältiger Kennzahlen quantifiziert werden. Im Schnitt ist jeder fünfte umgeschlagene Container in einem Seehafen ein Leercontainer. In Zeebrügge war im Jahr 2007 fast jeder dritte Container ein Leercontainer. Weltweit wurden im Jahr 2005 ca. 82 Millionen Leercontainern umgeschlagen. Die Gesamtkosten, die durch Leercontainer auf globaler Ebene im Jahr 2002 verursacht wurden, lassen sich auf 22,6 Mrd. US Dollar beziffern [Wan08]. Für das Jahr 2008 wurden diese bereits auf 33 Mrd. US Dollar geschätzt [Flä10]. Weiterhin lagern weltweit ca. 1,5 bis 2,5 Millionen Leercontainer in see- oder hafennahen Hinterlandgebieten. Diese bedingen einen hohen Platzbedarf und führen zur Verknappung der Lagerflächen in Seehäfen.

Im Jahr 2010 wurden im Hamburger Hafen - Deutschlands größter Containerhafen insgesamt knapp 7,9 Millionen Container umgeschlagen. Davon waren ca. 1,3 Mio. Leercontainer, wovon $44 \%$ seeseitig und $56 \%$ landseitig weitertransportiert wurden [Ham11]. So entstanden täglich geschätzt 3.200 Leercontainertransporte im Hamburger Raum. Diese Transporte bedingen ein hohes Verkehrsaufkommen, sind mitverantwortlich für Staus im Hafenbereich und führen $\mathrm{zu}$ hohen $\mathrm{CO}_{2^{-}}$und Lärmemissionen.

Das Wachstum in der Containerschifffahrt verschärft dieses Problem und führt ohne Prozessoptimierungen zwingend zu Kapazitätsanpassungen im Seeverkehr. Flächenerweiterungen für den Hafenbetrieb stoßen jedoch zunehmend auf Widerstand in der Bevölkerung und sind nur langfristig durchführbar. Ähnliches gilt für die Ausweitung der Containerverkehre. Negative Umweltauswirkungen und bestehende geographische Grenzen beschränken zusätzlich den Handlungsraum des Containerverkehrs. Die Optimierung der container-relevanten Logistikprozesse ist für Containereigner folglich wirtschaftlich, ökologisch und gesellschaftlich zwingend notwendig.

\subsection{Ursachen der Leercontainerproblematik}

In Abbildung 1 sind zwei über den Seeweg verbundene Regionen dargestellt. In jeder der beiden Regionen gibt es Importeure und Exporteure. Die Regionen betreiben Handel wodurch es zum Transport von Gütern in Seecontainern kommt. Es erfolgt somit ein Vollcontainerfluss von den Exporteuren der einen Region zu den Importeuren der anderen Region. Dadurch entsteht bei den Exporteuren ein Leercontainerbedarf und bei den Importeuren ein Leercontainerüberschuss. Es bilden sich Quellen und Senken für Leercontainer heraus. Die Notwendigkeit des Leercontainerflusses begründet sich daher unmittelbar aus dem Vollcontainerfluss. 


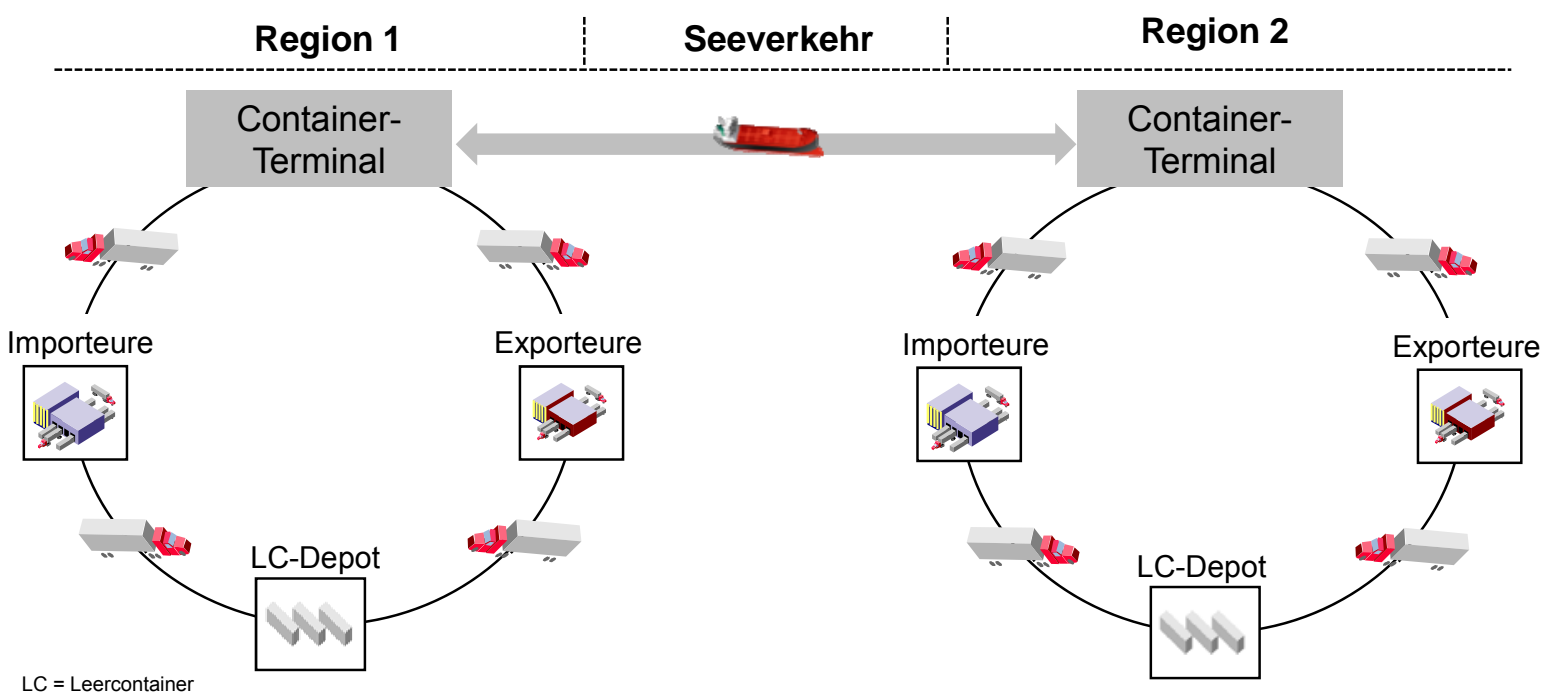

Abbildung 1: Vollcontainerfluss induziert Leercontainerfluss

Die einfachste Lösung der Leercontainerproblematik wäre ein direkter Leercontainertransport vom Überschuss- zum Bedarfsort innerhalb einer Region. Dieser Lösung stehen in der Containerschifffahrt hauptsächlich Disparitäten in verschiedenen Dimensionen (räumlich, zeitlich, kostenmäßig, qualitativ etc.) entgegen. Die Hauptursache der Leercontainerproblematik sind unpaarige Güterströme zwischen Import- und Exportregionen [Boi05] (räumlich-quantitative Disparität). Diese werden durch unterschiedliche, ggf. gegensätzliche, Import- und Exportvolumina der Handelsrelationen induziert, und stellen ein strukturelles Problem der Containerlogistik dar [The09]. Sie gehen auf unterschiedliche ökonomische Rahmenbedingungen, wie bspw. Industrialisierung, Industriestruktur, Konsumverhalten und Kaufkraft zurück [Wan08]. Auf globaler Ebene betrifft diese Thematik vor allem die wichtigsten Fahrtgebiete USA und Asien bzw. Europa und Asien. Zu den weiteren Gründen zählen die Entkopplung des Positionierungs- und Repositionierungsprozesses aufgrund notwendiger Zwischenlagerung der Leercontainer in Hafen- und Hinterlanddepots (Durchführung von Containerreinigung, -inspektion, -wartung und -instandsetzung), die räumliche Trennung und unterschiedliche Anzahl von Im- und Exporteuren, verschiedene Nachfragemenge der Im- und Exporteure, die Nachfrage unterschiedlicher Containerarten (40"-Standard, 20"-Standard, Reefer etc.), die Existenz unterschiedlicher Containereigner sowie unterschiedliche Kosten, Frachten und Qualitätsstandards.

\subsection{Stand der Forschung und Zielsetzung}

In der Wissenschaft sind einzelne Aspekte des Leercontainermanagements umfassend betrachtet. Die Ausführungen in der Literatur können in interne (unternehmensspezifische) und externe (unternehmensübergreifende) Ansätze kategorisiert werden. Weiterhin können strategische Herangehensweisen, 
informationstechnische und konstruktive Ansätze sowie mathematisch-formale Optimierungsmodelle differenziert werden [Voj09]. Vor allem in dem Bereich interner, mathematischer Optimierungsansätze findet sich eine Vielzahl von Publikationen. Einen Auszug zeigt, Tabelle 1.

\begin{tabular}{|c|c|c|c|}
\hline Autor & Schwerpunkt & Umfeld & Parameter/Problemgröße \\
\hline [Cho0] & $\begin{array}{l}\text { Fuzzy-basierte } \\
\text { Entscheidungsunterstützung }\end{array}$ & $\begin{array}{l}\text { Optimales Bestands-, Leasing- } \\
\text { und Repositionierungsmodell }\end{array}$ & $\begin{array}{l}6 \text { Häfen, } \\
5 \text { Schiffe }\end{array}$ \\
\hline [Ere09] & Robuste Optimierung & $\begin{array}{l}\text { Leercontainer- } \\
\text { Repositionierungs-Problem }\end{array}$ & $\begin{array}{l}20 \text { Häfen, } \\
600 \text { Tankcontainer }\end{array}$ \\
\hline [Fen08] & $\begin{array}{l}\text { Regionale } \\
\text { Repositionierungsstrategien }\end{array}$ & $\begin{array}{l}\text { Hafen-zu-Hafen- } \\
\text { Repositionierung }\end{array}$ & $\begin{array}{l}33 \text { Häfen, } \\
17 \text { Schiffe, } \\
3380 \text { 20“ Container (TEU) }\end{array}$ \\
\hline [Son08] & $\begin{array}{l}\text { Leercontainer- } \\
\text { Repositionierungs-Strategie }\end{array}$ & $\begin{array}{l}\text { Leercontainer- } \\
\text { Repositionierungs-Problem }\end{array}$ & $\begin{array}{l}3 \text { Häfen, } \\
1 \text { Schiff, } 500 \text { TEU }\end{array}$ \\
\hline [Lam07] & $\begin{array}{l}\text { Näherungsverfahren zur } \\
\text { Repositionierung }\end{array}$ & $\begin{array}{l}\text { Leercontainer- } \\
\text { Repositionierungs-Problem }\end{array}$ & $\begin{array}{l}3 \text { Häfen, } \\
9 \text { Schiffe }\end{array}$ \\
\hline [Li04] & $\begin{array}{l}\text { Bestandsreduzierung von } \\
\text { Leercontainern }\end{array}$ & Bestandsmodell für ein Hafen & $\begin{array}{l}1 \text { Hafen, } \\
100 \text { TEU }\end{array}$ \\
\hline [Li07] & $\begin{array}{l}\text { Bestandsreduzierung von } \\
\text { Leercontainern }\end{array}$ & $\begin{array}{l}\text { Bestandsmodell für mehrere } \\
\text { Häfen }\end{array}$ & $\begin{array}{l}3 \text { Häfen, } \\
226 \text { TEU }\end{array}$ \\
\hline [Che98] & $\begin{array}{l}\text { 2-Phasen Netzwerkmodell } \\
\text { (stochastisch) }\end{array}$ & $\begin{array}{l}\text { Leercontainer- } \\
\text { Repositionierungs-Problem }\end{array}$ & $\begin{array}{l}10 \text { Häfen, } \\
6 \text { Schiffe }\end{array}$ \\
\hline [Cra93] & $\begin{array}{l}\text { Leercontainer- } \\
\text { Repositionierungs-Strategie }\end{array}$ & $\begin{array}{l}\text { Leercontainer- } \\
\text { Repositionierungs-Problem }\end{array}$ & Keine \\
\hline
\end{tabular}

Tabelle 1: Stand der Forschung interner, mathematischer Optimierungsansätze (Auszug)

Diese Ansätze des Operations Research unterscheiden sich jeweils in der Zielfunktion, den Nebenbedingungen, den Lösungsalgorithmen sowie der Stochastizität der gegebenen Daten (Aufträge, Zeiten etc.). Jeder Ansatz orientiert dabei auf eine spezielle Problemformulierung, bspw. der Optimierung der Leercontainer-Repositionierung oder Leercontainerbestandsoptimierung, wobei die Vorteilhaftigkeit des Modells formal nachgewiesen wird. Die Optimierungsmodelle beschränken sich jedoch bei jedem der untersuchten Autoren auf einen Reeder und vernachlässigen kooperative Verhaltensweisen.

Kooperationen zwischen Reedereien und Containerleasinggesellschaften wurden bisher vor allem im Bereich organisatorischer Ansätze diskutiert [Voj10a, Boi06, Lop03]. Einen Ansatz zur kooperativen Neugestaltung des Leercontainermanagements in der maritimen Containerlogistik stellt die gemeinsame Nutzung eines Container-Pools durch mehrere Reedereien und Containerleasinggesellschaften dar. Dieses sogenannte Container-Pooling orientiert auf die Reduzierung des Repositionierungs-, Transport-, Umschlag- und Lagerungsaufwands durch gezielte Ausnutzung struktureller Ungleichgewichte [Voj09]. In der Praxis ist dieser Ansatz aufgrund unternehmenspolitischer Gründe (Container als Werbeträger) bisher kaum umgesetzt. Vor dem Hintergrund zunehmenden Kostendrucks und der Suche nach Möglichkeiten zu Effizienzsteigerungen im Leercontainermanagement werden zukünftig kooperative Gestaltungsansätze jedoch vermehrt an Bedeutung gewinnen [Voj10b, The09]. Für eine Optimierung und zur Unterstützung der operativen Planung sind mathematische Ansätze notwendig, die die verschiedenen Gestaltungsmöglichkeiten des Container- 
Poolings [Voj10b] modellseitig berücksichtigen sowie zwischen Pool-, Reeder- und Leasing-Containern unterscheiden. Hierzu werden derzeit in der Literatur keine Ansätze diskutiert.

Ziel dieses Beitrags ist daher die Vorstellung eines mathematischen Optimierungsmodells, das zum einen die Abläufe der maritimen Containerlogistik beschreibt und zum anderen den Einsatz des Container-Poolings berücksichtigt. Weiterhin ist es Ziel, die Einsparungspotentiale anhand eines beispielhaften Seeverkehrsnetzwerks zu quantifizieren.

Dazu werden im Folgenden die Grundfälle der Leercontainerlogistik beschrieben. Im Anschluss erfolgt die Modellierung eines Transportnetzwerks einer Reederei. Durch die Berücksichtigung des Container-Poolings werden anschließend mehrere Reeder in das Netzwerk integriert, deren Handlungsmöglichkeiten abgebildet und zusätzlich eine Unterscheidung zwischen Pool-, Reeder- und Leasing-Containern im Modell vorgenommen. Weiterhin erfolgt die mathematische Problemformulierung durch eine ganzzahlige lineare Optimierung. Anschließend wird eine Bewertung des ContainerPoolings sowie eine Zusammenfassung der Ergebnisse vorgenommen.

\section{Prozesse in der Leercontainerlogistik}

Zur Modellierung und Bewertung der maritimen Leecontainerlogistik müssen alle in einer Transportkette auftretenden Prozessfälle abgebildet werden, Abbildung 2.

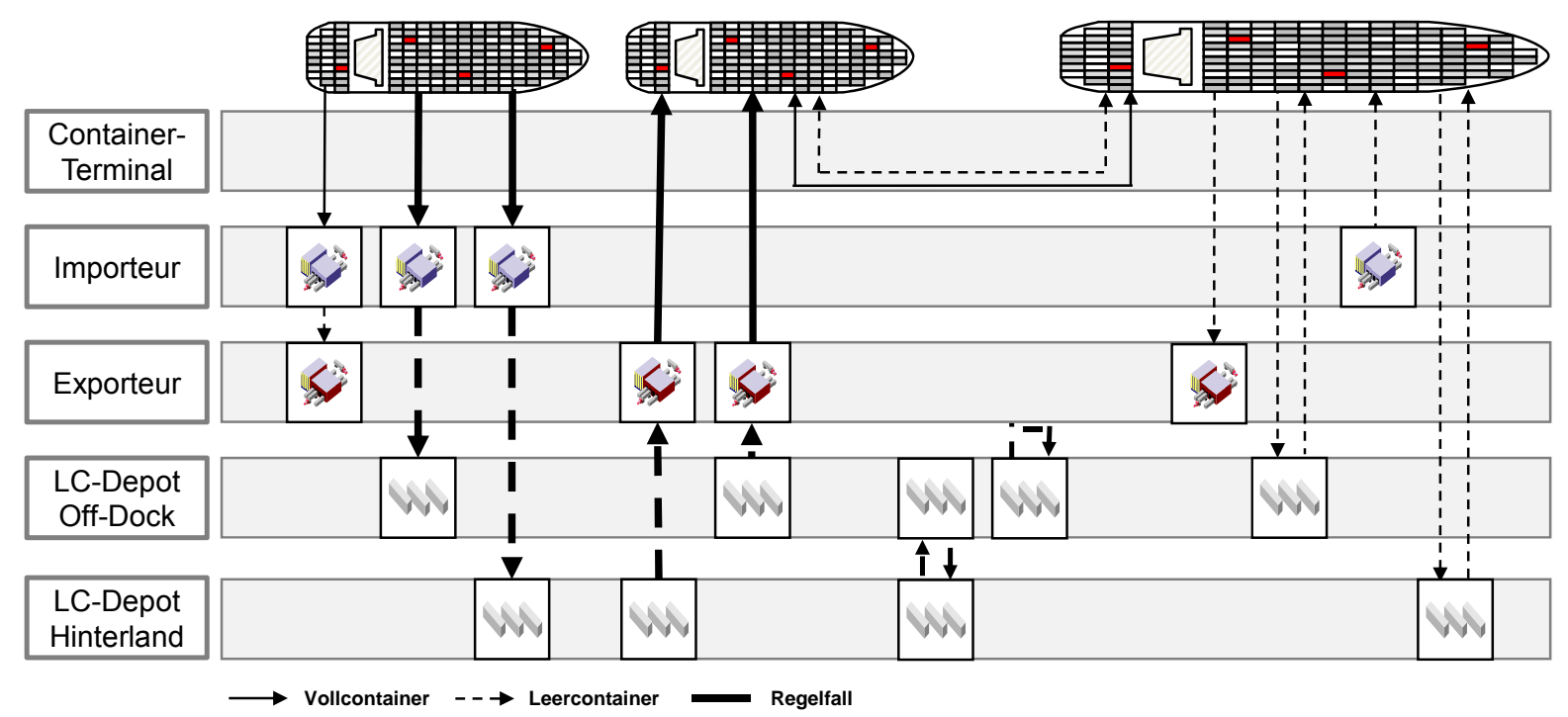

Abbildung 2: Grundfälle der maritimen Containerlogistik

Die in der Abbildung dargestellten roten Pfeile stellen die häufigsten Prozessfälle der Containerlogistik dar. Dabei werden Importcontainer einer Reederei zunächst von den Containerschiffen gelöscht, auf dem Container-Terminal zwischengelagert und später vom Importeur abgeholt („Merchant Haulage“) bzw. dem Importeur bereitgestellt („Carriers Haulage“). Nach dem der Container von dem Importeur entladen wurde, wird der Container je nach Möglichkeit und Vorgabe in ein Hafenoder Hinterlanddepot gebracht. Wenn ein Kunde einer Reederei einen Container für 
Exportware benötigt, wird ein Container aus einem Hafen- oder Hinterlanddepot organisiert und dem Exporteur bereitgestellt. Ist kein Reeder-Container vorhanden, stehen den Reedern verschiedene Leasingoptionen zur Verfügung. Nach erfolgter Beladung der Container organisiert in der Regel der Exporteur den Transport in das Container-Terminal. Dazu erhält er die genaue Bereitstellungszeit und den -ort von dem Reeder. Häufig werden in der Praxis auch Leercontainer zwischen Hafen- und Hinterlanddepots ausgetauscht. Das begründet sich aus der Reinigung und Prüfung der Leercontainer an zentraler Stelle. Hierzu wird in der Regel das im Hafen gelegene Containerdepot beansprucht. Im Fall des Transshipments werden Container in der Regel immer nur über das Container-Terminal umgeschlagen. In seltenen Fällen werden leere Container von Importeuren direkt an Exporteure übergeben. Die dargestellten Grundfälle bilden die Grundlage für die folgende Modellierung und Bewertung.

\section{$3 \quad$ Modellbildung}

Für die Optimierung der Leercontainerlogistik sind Modelle notwendig, die die Leercontainerbewegungen und -bestände sowie die damit verbundenen Kosten minimieren. Insbesondere zur Bestimmung der optimalen Bewegungen eines Seecontainers ist die Abbildung eines Transportnetzwerks erforderlich, welches mit Hilfe des Netzwerkflussproblems näher beschrieben werden kann.

In einem Seeverkehrsnetzwerk bilden alle für Reeder relevanten Standorte (Hinterland- und Off-Dock-Depots, Containerschiffe/-terminals, Ex- und Importeure etc.) zu jeder Planungsperiode eine Menge von Knoten. Zwischen diesen Knoten sind gerichtete Kanten definiert, die Handlungsalternativen des Lagerns, Transports (Bereitstellung, Repositionierung), Leasings, Off-Hires, Kaufs oder Verkaufs von Containern darstellen. Sie besitzen die Attribute Kapazität [untere Schranke d, obere Schranke u] sowie die Kosten c. Die Kapazitäten können im Fall einer Lagerung als Mindest- bzw. Maximalbestand oder bei einem Schiffstransport als Anzahl freier Slots interpretiert werden. Die Kosten sind auf einen Container bezogen, die bspw. durch Lagerung oder Transport entstehen.

\subsection{Modellierung aus Sicht eines Reeders}

Als Ausgangssituation zur Modellierung eines Seeverkehrsnetzwerks unter Berücksichtigung des Container-Poolings wird im Folgenden ein beispielhaftes maritimes Netzwerk für einen Reeder modelliert. Die Knoten bilden die relevanten Standorte (Depot, Terminal etc.). Es werden drei Planungsperioden angenommen. Alle Knoten werden in jede Planungsperiode dupliziert, um die dynamische Struktur des Netzwerks hinsichtlich jeglicher Operationsmöglichkeiten darzustellen. Im Ergebnis entsteht ein Zeit-Raum-Netzwerk, Abbildung 3. Die vertikalen und diagonalen Kanten spiegeln Transportoperationen wider; die horizontalen entsprechen einer Lagerung von einer in die nächste Periode. In den Kanten sind Reeder- und langfristig geleaste Container integriert abgebildet. 
Zusätzlich sind Containerströme abgebildet, die vor Beginn des betrachteten Planungszeitraumes im Netzwerk vorhanden sind bzw. nach der letzten Planungsperiode enden. Die Integration von Quellen q und Senken s ermöglicht die Modellierung von bspw. Anfangsbeständen in den Depots. Sie besitzen gleiche Kapazitätsschranken und keine Kosten, weil sie Anfangszustände von Containerbeständen und -flüssen repräsentieren. Für Container, die nach dem Planungszeitraum betrachtet werden, sind Kapazitätseinschränkungen und Kosten modellseitig nicht relevant.

Region 1

Region 2

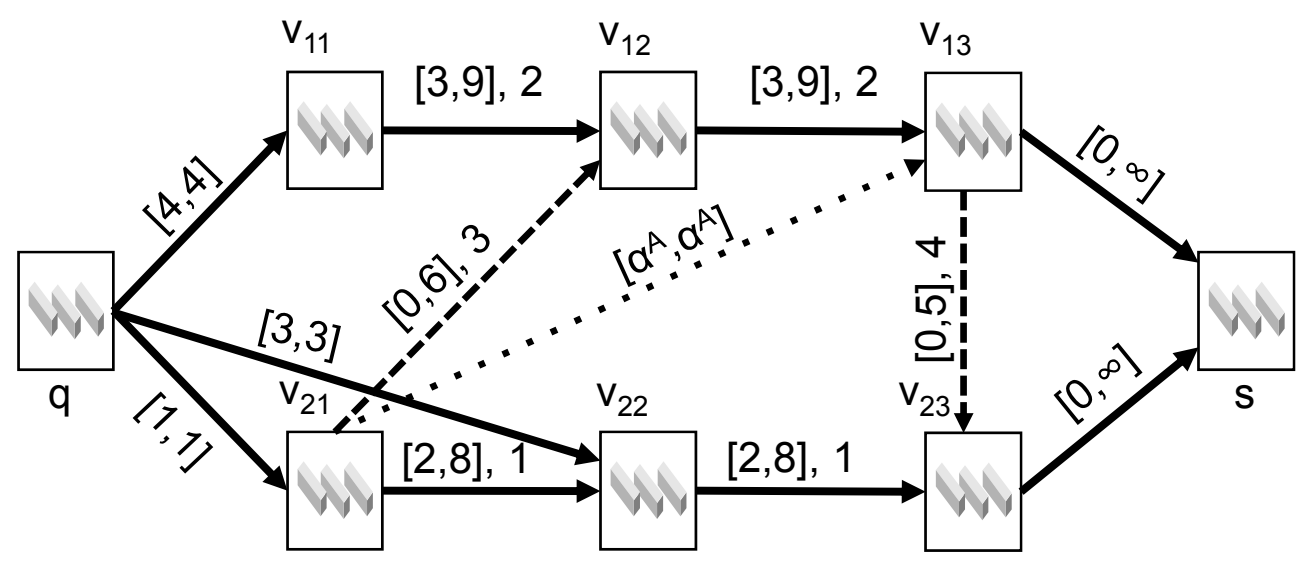

Periode

$\mathrm{T}_{1}$

$\mathrm{T}_{2}$

$\mathrm{T}_{3}$

$\longrightarrow$ Keine Ortsveränderung (Lagern)

$v_{i j}=$ Knoten $\mathrm{i}$ in Periode $\mathrm{j}$

$\rightarrow$ Ortsveränderung (Leercontainertransport)

... Ortsveränderung (Voll- und Leecontainertransport)

Abbildung 3: Zeit-Raum-Netzwerk einer Reederei

Jeder Auftrag A benötigt eine bestimmte Menge $\alpha^{A}$ an Leercontainern, die in einer definierten Planungsperiode an einem bestimmen Knoten vorhanden sein muss. Aus Sicht der Leercontainerlogistik fallen für die Abgabe der leeren Container in der Zielregion keine Kosten an, weil die die Leercontainer von einem Importeur in dem jeweils zugeordneten Depot bereitgestellt werden („Merchant Haulage“). Die Auftragskante $\left[\alpha^{A}, \alpha^{A}\right]$ entspricht somit gleichzeitig einem Voll- und einem Leercontainerfluss.

In der Betriebspraxis ist es häufig der Fall, dass im Transportnetzwerk nicht genügend Container zur Bedarfsdeckung vorhanden sind. In dem Fall können Reedereien auf weitere Leasingcontainer zurückgreifen. Alternativ können Reeder kurzfristig gemietete Container "off-leasen“. Diese sind im Modell explizit gekennzeichnet. Dazu wird in jeder Periode $t$ ein Leasingknoten $L_{t}$ eingefügt. Von diesen Knoten führen Kanten mit der Kapazität $\left[0, \mathrm{u}_{\llcorner}{ }^{\vee}\right]$ zu allen Standorten des Reeders, wobei $\mathrm{u}_{\llcorner}{ }^{v}$ die maximale Anzahl an Containern entspricht, die ein Reeder von der Leasinggesellschaft erhalten kann, Abbildung 4. 


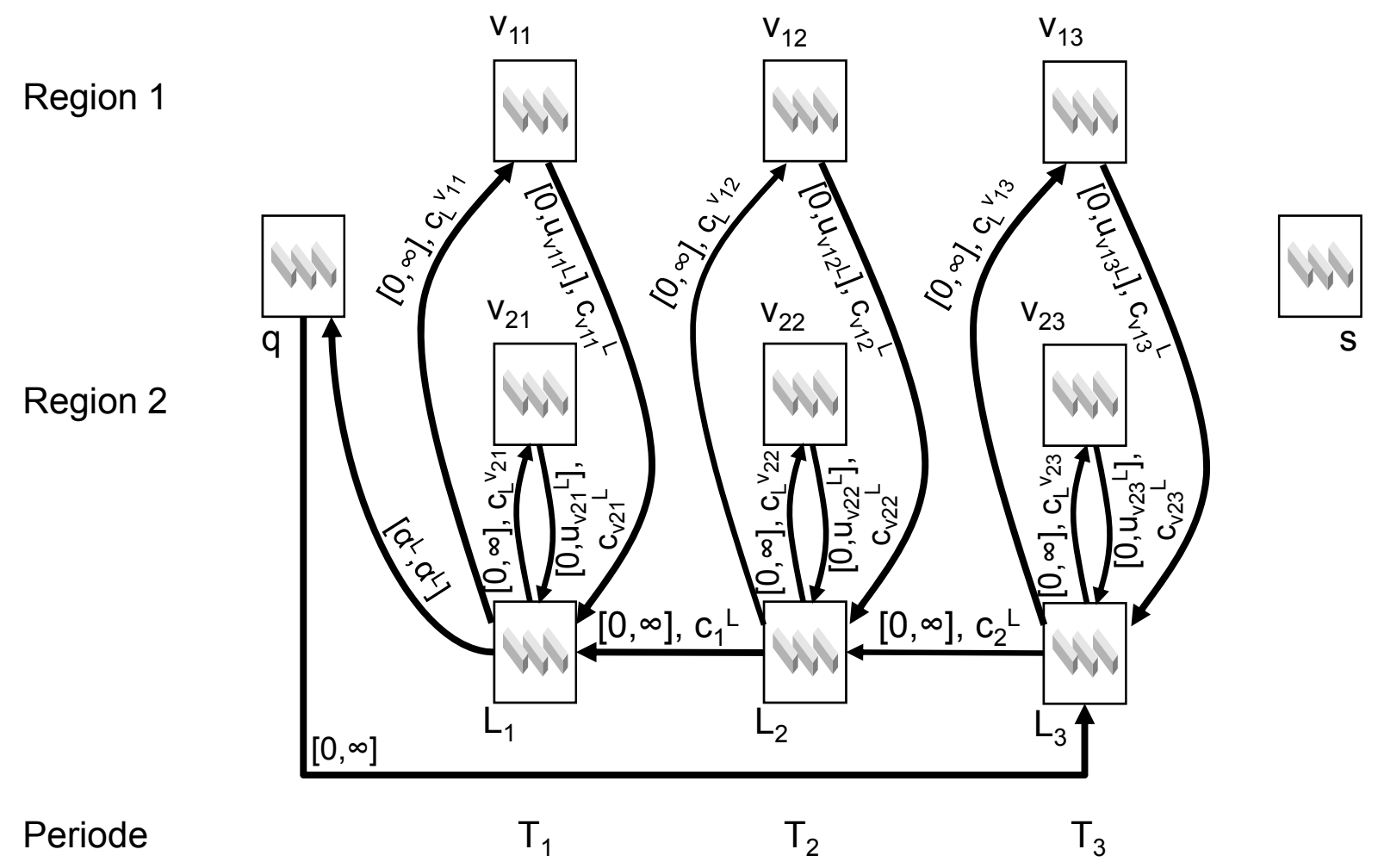

Abbildung 4: Einbindung des kurzfristigen Leasings im Zeit-Raum-Netzwerk einer Reederei

Die Mietkosten sollen zeitabhängig berechnet werden. Hierfür ist es notwendig, eine Menge $\alpha^{L}$ von dem ersten Leasingknoten zur Quelle zurückzuführen. Diese Menge entspricht der Anzahl Leasingcontainer, die zu Beginn des Planungszeitraums im Bestand der Reederei ist. Zusätzlich werden Kanten von $L_{t+1} z u L_{t}$ mit der Kapazität $[0, \infty]$ hinzugefügt. Die Kosten $c_{t}{ }^{L}$ dieser Kanten entsprechen den Leasingkosten über den Zeitraum von $\mathrm{t} z \mathrm{u} t+1$. Wird bspw. ein Container in der Periode $t$ geleast und in der Periode $t+2$ zurückgegeben, ergeben sich die Kosten aus $C_{t+1}{ }^{L}+C_{t}{ }^{L}$. Zur Sicherstellung der Flusserhaltung muss weiterhin eine neue Kante von der Quelle zu dem Leasingknoten der letzten Periode führen. Der resultierende Fluss entspricht den noch nicht zurückgegebenen Leasingcontainern. Schließlich muss im Sinne der Absicherung der Lösbarkeit des Problems die Möglichkeit zur Aussonderung von Containern gegeben sein. Das ist insbesondere dann der Fall, wenn die Summe der oberen Kapazitätsschranken nicht den gesamten Containerbestand abdeckt und somit das Netzwerk keinen zulässigen Fluss aufweist. Dazu wird von jedem Knoten eine kapazitiv unbegrenzte Kante zur Senke geführt, wobei hohe Strafkosten $c^{P}$ angesetzt werden, um diese Option möglichst unattraktiv für Reeder zu gestalten. In der Abbildung wird aus Gründen der Übersichtlichkeit bewusst darauf verzichtet.

\subsection{Modellierung des Container-Poolings}

Durch die Berücksichtigung des Container-Poolings werden mehrere Reeder in das Netzwerk integriert und deren Handlungsmöglichkeiten abgebildet. Eine mögliche Variante des Container-Poolings ist die partielle Teilnahme von Reederein und Leasinggesellschaften an einen Pool. Dabei wird nur ein Teil des gesamten (c) 2011 Logistics Journal : Proceedings - ISSN 2192-9084 
Containerbestands der Beteiligten in den Pool integriert, während die Unternehmen eigene Container im Bestand halten. In der hier gewählten Variante nehmen zwei Reedereien und eine Leasinggesellschaft an einem Container-Tausch teil, wobei die Leasinggesellschaft an beide Reeder auch Leasingcontainer bereitstellen kann. Für dieses Beispiel werden somit Pool-, Reeder- und Leasingcontainer in einem Netzwerk modelliert. Weiterhin wird angenommen, dass mind. ein Depot des jeweiligen Reeders in räumlicher Nähe zu dem des anderen ist. Zusätzlich wird angenommen, dass die Reedereien ihre Container in einem dienstleisterbetriebenen Depot lagern, so dass für beide gleiche Depotkosten pro Einheit entstehen. Die Nutzungsgebühren für eigene Container sind für jeden Reeder gleich, während die Nutzungsgebühren von Pool-Containern mit einem zu vereinbarenden Zuschlag kalkuliert werden. Die Zusammenführung der jeweiligen Transportnetzwerke zeigt Abbildung 5, wobei die Standorte $\mathrm{I}_{\mathrm{A}}^{3}$ und $\mathrm{I}_{\mathrm{B}}^{3}$ als gemeinsames Depot $\mathrm{I}_{\mathrm{AB}}^{3}$ zusammengelegt wurden.

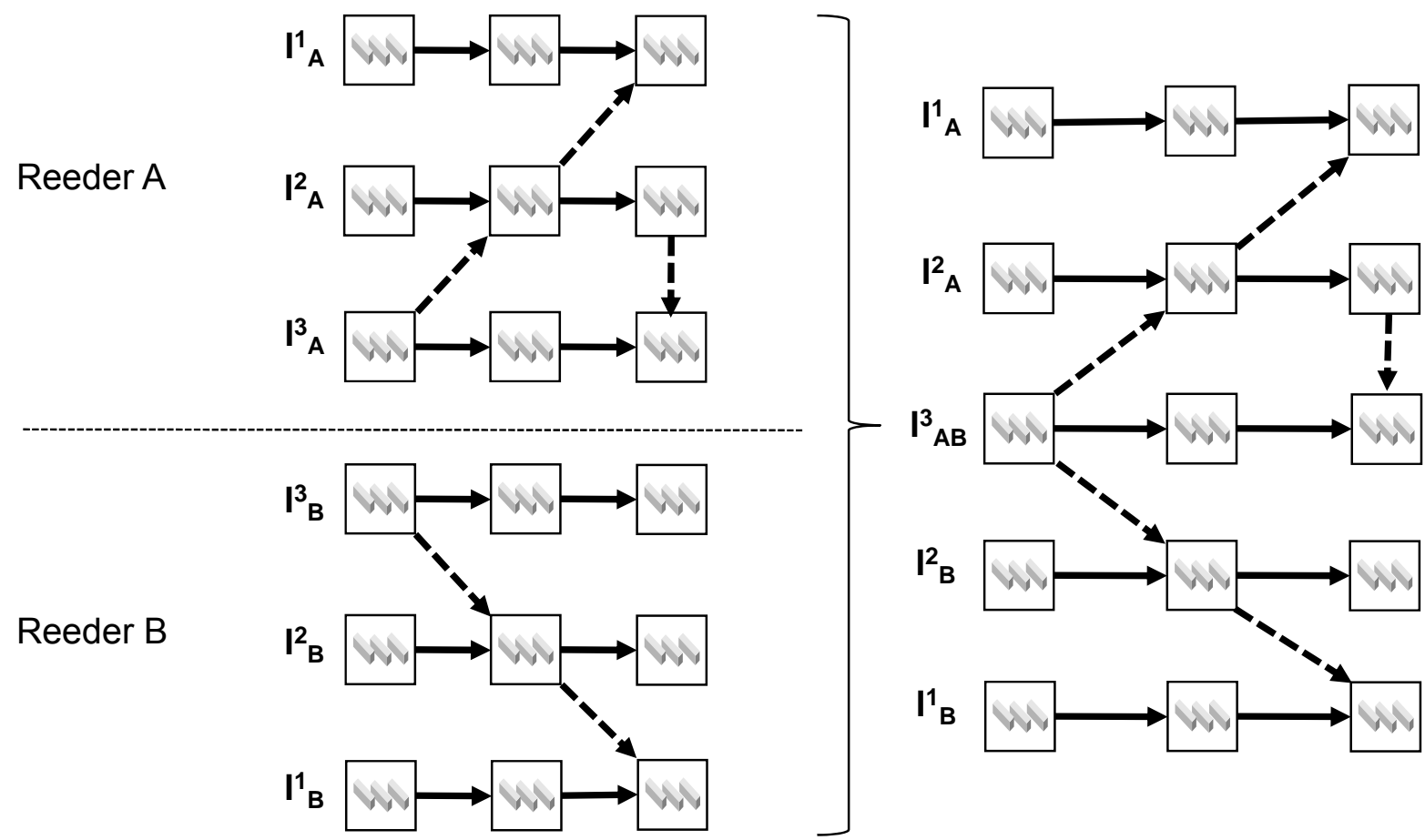

"Abbildung 5: Zusammenführung der Transportnetzwerke zweier Reedereien

Darüber hinaus muss zur Abbildung der verschiedenen Container und den dazugehörigen Eigentümern das Modell erweitert werden. Die Besonderheit besteht darin, dass die Reeder auf ihren Relationen entweder ihre eigenen, Pool- oder Leasing-Container, jedoch nicht die Container der anderen Reederei verwenden dürfen. Im Modell sind daher Kanten so zu deklarieren, dass nur bestimmte Containertypen eingesetzt bzw. ausgewählte Typen ausgeschlossen werden. Weiterhin müssen im Sinne der Flusserhaltungsbedingung zusätzlich Anfangs- und Endknoten integriert werden. 
Auf den Anfangskanten sind die Containertypen ebenfalls zu differenzieren, weil der Anfangsbestand einer Containerart für jeden Ort bekannt ist. Beispielsweise dürfen keine Container vom Reeder A auf eigenständigen Kanten des Reeder B gehandhabt werden. Auf den Abgangskanten sind alle Container erlaubt, weil diese nicht die Planung beeinflussen. Einzige Ausnahme stellen Leasingcontainer dar, weil deren Abgabezeitpunkt häufig vorher bekannt ist. Auf die Darstellung kapazitiv unbegrenzter Kanten zur Senke (Bsp.: Aussonderung), der Leasingknoten und der Attribute der jeweiligen Kanten wurde bewusst verzichtet, Abbildung 6.

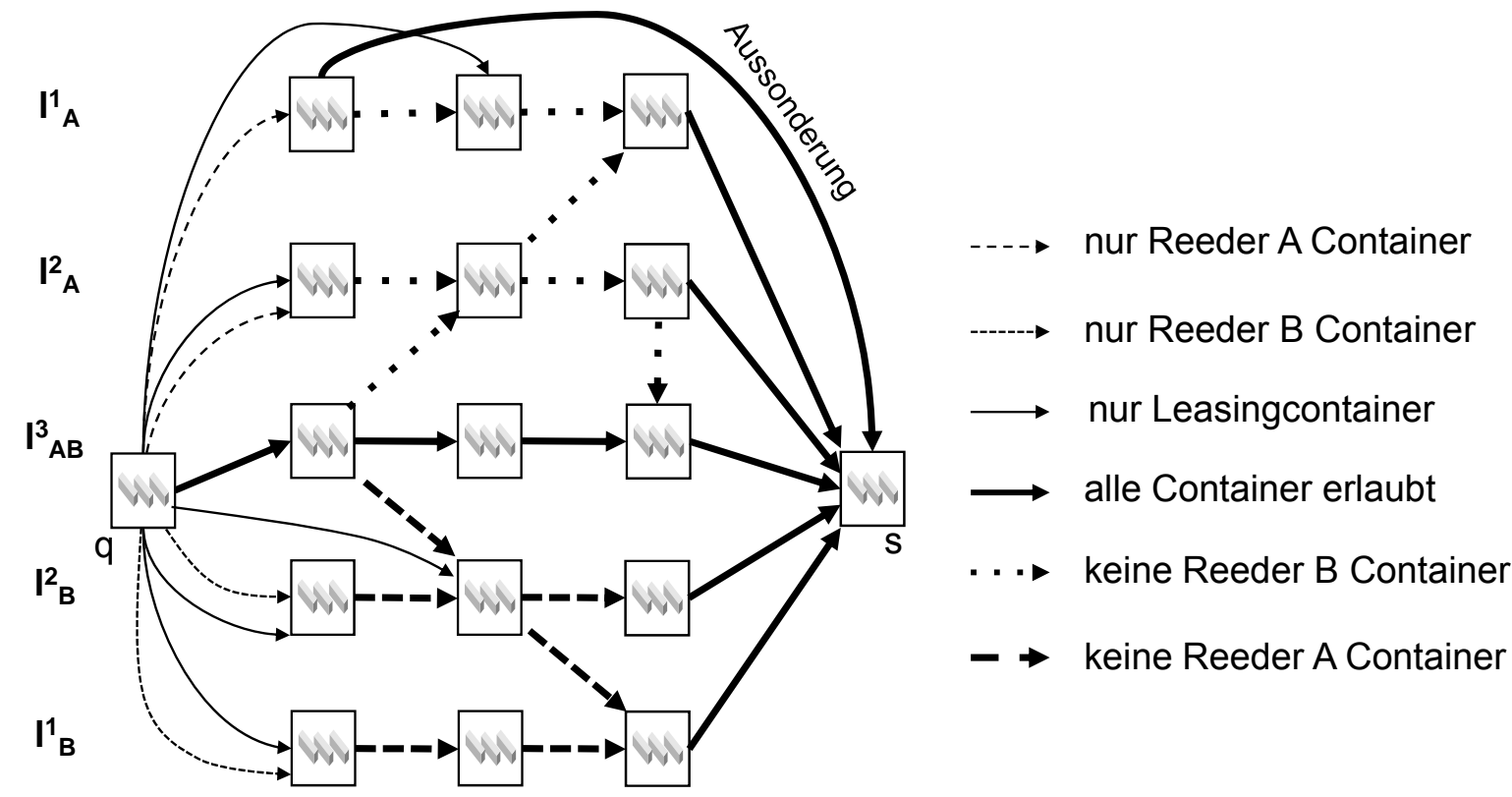

$\begin{array}{llll}\text { Periode } & T_{1} & T_{2} & T_{3}\end{array}$

Abbildung 6: Seeverkehrsnetzwerk-Modell unter Berücksichtigung von Container-Pooling

Auf Basis dieses schematischen Modells kann die Formulierung eines mathematischen Modells erfolgen.

\subsection{Mathematische Formulierung}

Für die Optimierung von Leercontainerströmen wird im Folgenden ein allgemeines mathematisches Modell für das Container-Pooling formuliert. Das Modell berücksichtigt die Beteiligung beliebig vieler Reeder und Leasinggesellschaften. Gleichgestellt mit dem obigen Beispiel, besitzen die Teilnehmer am Pool weiterhin eigene Container. Aufgrund dieses Sachverhaltes sowie der damit einhergehenden Erlaubnisbedingung für Container auf bestimmten Kanten, kann das Optimierungsproblem nicht auf Basis eines Minimumkosten-Netzwerkflussproblems gelöst werden. Stattdessen wird ein ganzzahliges lineares Optimierungsproblem zu Grunde gelegt:

Für jeden Reeder existiert ein Netzwerk $N=(V, E, q, s, u)$. Das Netzwerk besteht aus einer Menge $V$ Knoten und einer Menge $E$ Kanten. Für eine Kante $\varepsilon=\varepsilon^{-} \varepsilon^{+} \in E$ ist 
$\varepsilon^{-}$der Anfangsknoten und $\varepsilon^{+}$der Endknoten. Die Knoten $q \in V$ und $s \in V$ werden als Quelle bzw. Senke und $u$ als obere Kapazitätsfunktion bezeichnet. Das Netzwerk hat zusätzlich eine untere Kapazitätsfunktion $d$ und eine Kostenfunktion $c$. Die Menge $R:=\left\{r_{1}, r_{2}, \ldots, r_{n}\right\}$ beinhaltet $n$ Reeder als Pool-Teilnehmer.

Ein Fluss $f$ im Netzwerk $N$ ist eine Funktion mit den Eigenschaften:

$$
\begin{array}{lll}
0 \leq f(\varepsilon) \leq u(\varepsilon), & \forall \varepsilon \in E & \text { (Kapazitätsbedingung) } \\
\sum_{\varepsilon^{-}=v} f(\varepsilon)=\sum_{\varepsilon^{+}=v} f(\varepsilon), & \forall v \in V \backslash\{q, s\} &
\end{array}
$$

Für das Container-Pooling muss ein Mengensystem P eingeführt werden, dass alle für das Modell relevanten Container berücksichtigt:

$\mathrm{P}:=\left\{B_{P} \mid b_{p} \in B_{P}\right.$, ist Pool-Container $\mathrm{p}$, an dem alle $r \in R$ Nutzerrechte haben $\} \cup$ $\left\{B_{R} \mid b_{r_{i}} \in B_{R}\right.$, ist Reeder - Container, an dem $r_{i} \in R$ Nutzerrechte hat $\} \cup$ $\left\{B_{L} \mid b_{l} \in B_{L}\right.$, ist geleaster Container $\mathrm{I}$, an dem alle $r \in R$ Nutzerrechte haben $\}$

Enthalten sind dabei die Reeder-Container, die Pool-Container sowie die Leasingcontainer.

Die Netzwerke der Reedereien werden im ersten Schritt durch eine umfangreiche Kontraktion der Kanten und Knoten zusammengelegt. Im Ergebnis sei $E_{\text {Pool }}$ die Kantenmenge, $V_{P \circ o l}$ die Knotenmenge und $u_{P \circ o l}$ die neue obere Kapazitätsfunktion des verschmolzenen Netzwerks $N_{\text {Pool }}$ mit $N_{\text {Pool }}:=\left(V_{\text {Pool }}, E_{\text {Pool }}, q, s, u_{\text {Pool }}\right)$. Zusätzlich sind $d_{\text {Pool }}$ die neue obere Kapazitätsfunktion und $c_{\text {Pool }}$ die neue Kostenfunktion. Zur Differenzierung der Containerrechte ist $f_{P}(\varepsilon)$ der Fluss des Containers $P \in \mathrm{P}$ auf der Kante $\varepsilon$.

Die Optimierung des Netzwerks erfolgt mit folgendem ganzzahligen linearen Optimierungsproblem unter Berücksichtigung der erlaubten Containerflüsse und den jeweils anfallenden Kosten. Das dargestellte Modell kann anschließend rechnergestützt implementiert und für ein praktisches Beispiel angewandt werden.

$$
\begin{aligned}
& \sum_{P \in \mathbb{P}} \sum_{\varepsilon \in E_{\text {Pool }}} f_{P}(\varepsilon) c_{\text {Pool }}(\varepsilon) \rightarrow \min \\
& \sum_{P \in \mathrm{P}} \sum_{\varepsilon^{-}=v} f_{P}(\varepsilon)=\sum_{P \in \mathrm{P}} \sum_{\varepsilon^{+}=v} f_{P}(\varepsilon), \quad \forall v \in V_{\text {Pool }} \backslash\{q, s\} \\
& d_{\text {Pool }}(\varepsilon) \leq \sum_{P \in \mathrm{P}} f_{P}(\varepsilon) \leq u_{\text {Pool }}(\varepsilon), \forall \varepsilon \in E_{\text {Pool }} \\
& f_{P}(\varepsilon)=0, \forall \varepsilon \notin\{\text { erlaubt Kanten für Container } P\} \\
& f_{P}(\varepsilon) \geq 0 \text { und ganzzahlig, } \quad \forall P \in \mathrm{P}, \quad \forall \varepsilon \in E_{\text {Pool }} \\
& \text { (Flusserhaltungsbedingung) } \\
& \text { (Kapazitätsbedingung) } \\
& \text { (Erlaubnisbedingung) } \\
& \text { (Positiv-/Ganzzahligkeits-Bed.) }
\end{aligned}
$$




\section{$4 \quad$ Bewertung des Rationalisierungspotentials des Container-Poolings}

Zur Bewertung der Einsparungspotentiale durch den Einsatz des Container-Poolings wurde ein fiktives Netzwerk zweier Reedereien in einem Simulationsmodell abgebildet. Die Reedereien sind durch eine unterschiedliche Größe ihrer Containerflotten und unterschiedlicher Relationen charakterisiert. Für die Durchführung zahlreicher Simulationsstudien wurde eine Parametermaske erstellt, in der Anfangsbestände, Export- und Importspannen, die Länge einer Planungsperiode, Kapazitätsschranken und Kosten definiert werden können. In jeweils 30 Studien wurden die Transportnetzwerke der Reeder mit bzw. ohne Anwendung des Container-Poolings simuliert. Dazu wurden jeweils 52 Wochen gewählt und folgende realitätsnahe Kostenwerte angenommen: Leasingkosten \$200/Periode, Transportkosten $\$ 800 /$ Repositionierung, Lagerkosten von $\$ 20$ / Periode, Ein- und Auslagerungskosten $\$ 100$ / Container und Terminal Handling Charge $\$ 50 /$ Periode. Zudem wurden alle Container aus dem vorherigen Bestand beider Reeder zu einem Pool-Containerbestand zusammengeführt. Weiterhin wurden aufgrund der unterschiedlichen Containerflottengröße der Reedereien und im Sinne einer Gleichberechtigung beider Partner die Nutzungsgebühren pro Container und Periode der Pool-Container unterschiedlich hoch definiert. Die resultierenden, durchschnittlichen Kostenverläufe sind in Abbildung 7 dargestellt.

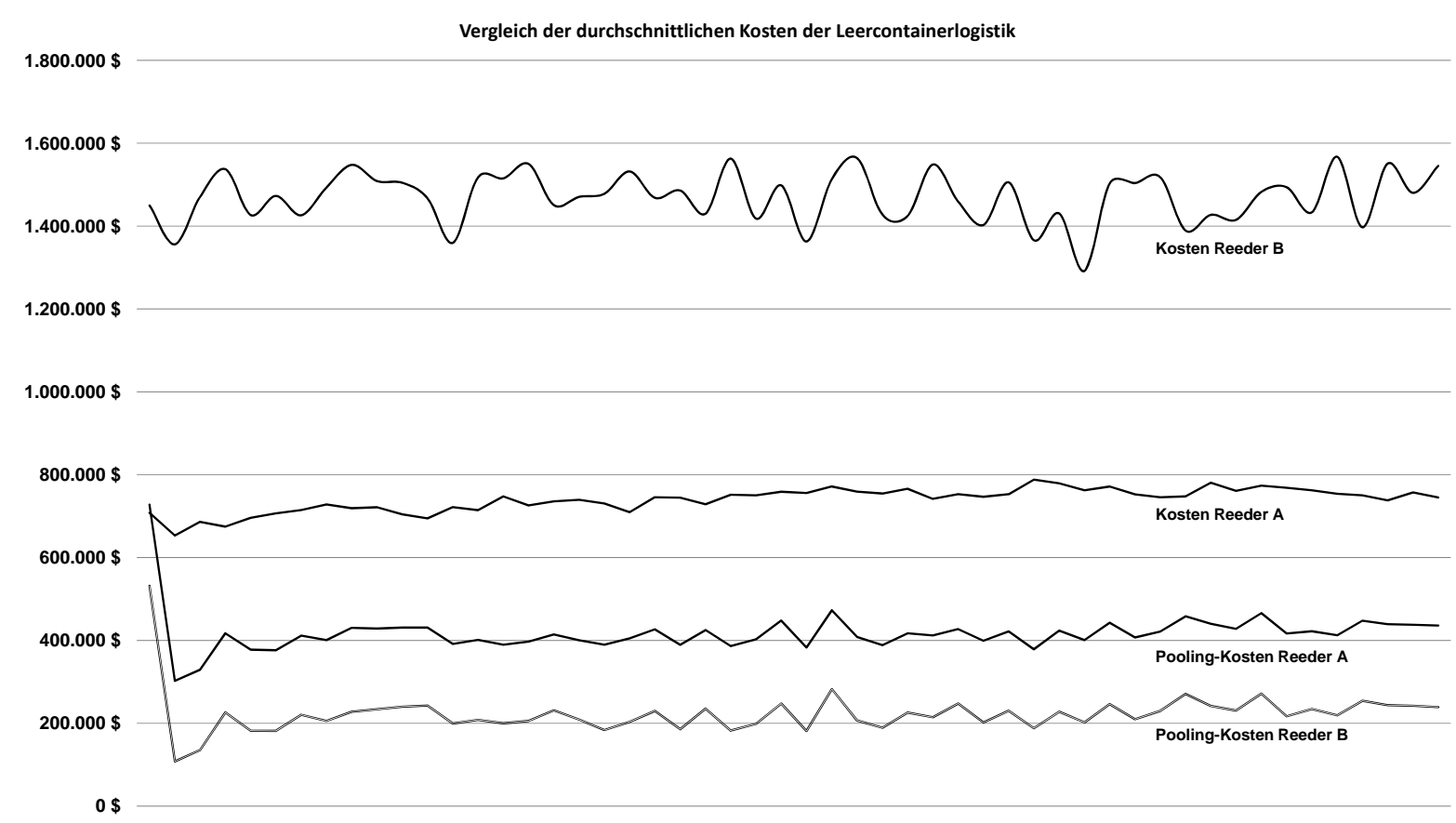

Abbildung 7: Auswertung von Simulationsstudien mit/ohne Container-Pooling

Auf Basis der zu Grunde gelegten Daten können beide Reeder $70 \%$ durch die gemeinsame Einbringung ihrer Containermenge in einen Pool einsparen. Durch das Pooling entfallen fast vollständig Repositionierungen. Diese begründen als größter Kostenfaktor das sehr hohe Einsparungspotential. Gleichzeitig können freie Slots auf den Containerschiffen für Vollcontainer verwendet werden. 


\section{$5 \quad$ Zusammenfassung}

Leercontainerungleichgewichte stellen ein strukturelles Problem der maritimen Containerlogistik dar. Ein uneffektives und ineffizientes Leercontainermanagement verursacht hohe Kosten und ist mitverantwortlich für die Entstehung unnötiger $\mathrm{CO}_{2}$ und Lärmemissionen. Reedereien und Containerleasinggesellschaften schöpfen aufgrund unternehmenspolitischer Gründe noch immer nicht alle kooperationsbasierten Potentiale zur Optimierung und Rationalisierung der Logistikprozesse aus. Daher war es das Ziel dieses Beitrags, potentielle Kosteneinsparungen durch den Einsatz des Container-Poolings im Vergleich zum unkooperativen Verhalten aufzuzeigen.

Dem dargestellten Modell unterliegen jedoch vielfältige Annahmen, die umfassendes Wissen über alle Gegebenheiten voraussetzen. In der Realität ist dieser Sachverhalt in der Regel nicht gegeben. Deshalb sollte bspw. durch dynamische Programmierung, eine geeignete Wahl des Planungshorizonts sowie eine kürzere Länge der Planungsperioden den stochastischen Einflüssen und Unsicherheiten entgegengewirkt werden.

\section{Literatur}

[Boi05] Boile, M.: Empty Marine Container Management in a Port MegaCity Region, New York Metropolitan Transport Council, Presentation, 19.10.2005

[Boi06] Boile, M.; Abookaber, N.: Empty Intermodal Container Management Report, Rutgers University, 2008

[Che98] Cheung, R.K; Chen, C.Y.: A two-stage stochastic network model and solution methods for the dynamic empty container allocation problem, Transportation Science, 32(2), 1998, S. 142-162

[Cho10] Chou, C.-C.; Gou, R.-H.; Tsai, C.-L.; et al: Application of a mixed fuzzy decision making and optimization programming model to the empty container allocation, Applied Soft Computing, 10, 2010, S. 1071-1079

[Cra93] Crainic, T. G.; Gendreau, M.; Dejax, P.: Dynamic and Stochastic Models for the Allocation of Empty Containers, in: Operations Research 41(1), 1993, S. $102-126$

[Ere09] Erera, A. L.; Morales, J. C.; Savelsbergh, M.: Robust Optimization for Empty Repositioning Problems, Operations Research, 57(2), 2009, S. 468-483

[Fen08] Feng, C.-M; Chang, C.H.: Empty container reposition planning for intraAsia liner shipping, Maritime Policy \& Management, 35(5), 2008, S. 469489 
[Flä10] Flämig, H.; Herz, N.; Wolff, J.: Managing empty containers - Folding boosts efficiency, in: Baltic Transport Journal, 4, 2010, S. 40-41

[Ham11] Hafen Hamburg: Containerumschlag 2010, http://www.hafenhamburg.de/sites/default/files/images/daten/time-ser-con_10.xls, Abrufdatum: 16. März 2011

[Lam07] Lam, S.-W.; Lee, L.-H.; Tang, L.-C.: An approxiamte dynamic programming approach for the empty container allocation problem, Transportation Research Part C, 15, 2007, S.265-277

[Li04] Li, J.-A.; Liu, K.; Leung, S. C. H.; Lai, K. K.: Empty container management in a port with long-run average criterion. Mathematical and Computer Modeling, 40(1-2), 2004, S. 85-100

[Li07] Li, J.-A.; Leung, S.; Wu, Y.; Liu, K.: Allocation of empty containers between multi-ports, European Journal of Operational Research, 182, 2007, S. 400-412

[Lop03] Lopez, E.: How do ocean carrier organize the empty container repositioning activity in the USA?, Maritime Policy \& Management, 30(4), 2003, S. 339-355

[Son08] Song, D.-P.; Dong, J.-X.: Empty container management in cyclic shipping routes, Maritime Economics \& Logistics, 10(4), 2008, S. 335361

[The09] Theofanis, S.; Boile, M.: Empty marine container logistics: facts, issues and management strategies, GeoJournal: 74(1), 2008, S. 51-65

[Voj09] Vojdani, N.; Rajchowski, R.; Lootz, F.; Pickel, M.: Containerpooling: Optimierung der Leercontainerbereitstellung in Seehäfen, Tagungsband, 5. Fachkolloquium der WGTL, IImenau, 01.-02. Oktober 2009, S. 271 280

[Voj10a] Vojdani, N; Lootz, F., Rajchowski, R.: Maritime Logistik - Lösungsansätze zur Optimierung der Leercontainerbereitstellung in Seehäfen, Jahrbuch Logistik, Korschenbroich: free beratung $\mathrm{GmbH}$, 2010, S. 149-153

[Voj10b] Vojdani, N.: Optimierung der Leercontainerbereitstellung in Seehäfen mittels Container-Pooling, Tagungsband, 10. Rostocker Logistik Forum - Innovationen in der Logistik, Rostock, 23. - 24. Juni 2010, S. 1-12

[Wan08] Wang, R.; Zhao, X.; Yu, W.; Zou, W.: The study on empty container allocation in the container transportation, Singapure: Industrial Engineering and Engineering Management, 2008 